\title{
Front Matter: Volume 6896
}

, "Front Matter: Volume 6896," Proc. SPIE 6896, Integrated Optics: Devices, Materials, and Technologies XII, 689601 (11 March 2008); doi:

$10.1117 / 12.791603$

SPIE Event: Integrated Optoelectronic Devices 2008, 2008, San Jose, California, SPIE. United States 


\title{
PROCEEDINGS OF SPIE
}

\section{Integrated Optics: Devices, Materials, and Technologies XII}

\author{
Christoph M. Greiner \\ Christoph A. Waechter \\ Editors \\ 21-23 January 2008 \\ San Jose, California, USA \\ Sponsored and Published by \\ SPIE
}

Volume 6896 
The papers included in this volume were part of the technical conference cited on the cover and title page. Papers were selected and subject to review by the editors and conference program committee. Some conference presentations may not be available for publication. The papers published in these proceedings reflect the work and thoughts of the authors and are published herein as submitted. The publisher is not responsible for the validity of the information or for any outcomes resulting from reliance thereon.

Please use the following format to cite material from this book:

Author(s), "Title of Paper," in Integrated Optics: Devices, Materials, and Technologies XII, edited by Christoph M. Greiner, Christoph A. Waechter, Proceedings of SPIE Vol. 6896 (SPIE, Bellingham, WA, 2008) Article CID Number.

ISSN 0277-786X

ISBN 9780819470713

Published by

SPIE

P.O. Box 10, Bellingham, Washington $98227-0010$ USA

Telephone +1 3606763290 (Pacific Time) · Fax +1 3606471445

SPIE.org

Copyright (c) 2008, Society of Photo-Optical Instrumentation Engineers

Copying of material in this book for internal or personal use, or for the internal or personal use of specific clients, beyond the fair use provisions granted by the U.S. Copyright Law is authorized by SPIE subject to payment of copying fees. The Transactional Reporting Service base fee for this volume is $\$ 18.00$ per article (or portion thereof), which should be paid directly to the Copyright Clearance Center (CCC), 222 Rosewood Drive, Danvers, MA 01923. Payment may also be made electronically through CCC Online at copyright.com. Other copying for republication, resale, advertising or promotion, or any form of systematic or multiple reproduction of any material in this book is prohibited except with permission in writing from the publisher. The CCC fee code is 0277-786X/08/\$18.00.

Printed in the United States of America.

Publication of record for individual papers is online in the SPIE Digital Library.

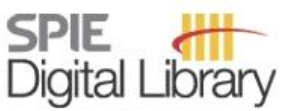

SPIEDigitalLibrary.org

Paper Numbering: Proceedings of SPIE follow an e-First publication model, with papers published first online and then in print and on CD-ROM. Papers are published as they are submitted and meet publication criteria. A unique, consistent, permanent citation identifier (CID) number is assigned to each article at the time of the first publication. Utilization of CIDs allows articles to be fully citable as soon they are published online, and connects the same identifier to all online, print, and electronic versions of the publication. SPIE uses a six-digit CID article numbering system in which:

- The first four digits correspond to the SPIE volume number.

- The last two digits indicate publication order within the volume using a Base 36 numbering system employing both numerals and letters. These two-number sets start with $00,01,02,03,04,05$, $06,07,08,09,0 \mathrm{~A}, 0 \mathrm{~B} \ldots \mathrm{OZ}$, followed by 10-1Z, 20-2Z, etc.

The CID number appears on each page of the manuscript. The complete citation is used on the first page, and an abbreviated version on subsequent pages. Numbers in the index correspond to the last two digits of the six-digit CID number. 


\section{Contents}

ix Conference Committee

SESSION 1 MODELING

689602 Advanced FEM analysis of optical waveguides: algorithms and applications (Invited Paper) [6896-01]

F. Schmidt, S. Burger, J. Pomplun, L. Zschiedrich, Zuse-Institute Berlin (Germany) and JCMwave GmbH (Germany)

689603 Coupled optical defect microcavities in 1D photonic crystals and quasi-normal modes [6896-02]

M. Maksimovic, M. Hammer, E. van Groesen, Univ. of Twente (Netherlands)

689604 Optimal design of vertically emitting circular Bragg disk resonator lasers [6896-03]

X. Sun, California Institute of Technology (USA); J. Schever, Tel-Aviv Univ. (Israel); A. Yariv, California Institute of Technology (USA)

689605 Thermal radiation antennas made of multilayer structures containing negative index metamaterials [6896-04]

M. Maksimovic, M. Hammer, Univ. of Twente (Netherlands); Z. Jaksic, Univ. of Belgrade (Serbia and Montenegro)

\section{SESSION 2 GLASS WAVEGUIDES AND DEVICES}

689606 Hybrid $\mathrm{Nd}^{3+}$-doped passively Q-switched waveguide laser made by ion exchange [6896-06]

R. Salas-Montiel, L. Bastard, J.-E. Broquin, IMEP, Lab. d'Hyperfréquence et de Caractérisaton, INP Grenoble (France)

689607 Optical waveguide gratings in chalcogenide glass [6896-07]

X. Xia, M. Solmaz, H. Park, X. Cheng, C. K. Madsen, Texas A\&M Univ. (USA)

689608 Realization of $\mathrm{Ag}^{+} / \mathrm{Na}^{+}$ion-exchanged surface and buried waveguides on germanate glasses [6896-08]

J. Grelin, E. Ghibaudo, J.-E. Broquin, IMEP-LAHC, MINATEC-INPG (France)

\section{SESSION 3 WG ENGINEERING AND NANOFABRICATION}

6896 OB Ultra-compact polymer and silicon modulator design based on photonic crystal ring resonators [6896-13]

Z. Qiang, W. Zhou, Univ. of Texas at Arlington (USA); R. A. Soref, Air Force Research Lab. (USA); Z. Ma, Univ. of Wisconsin, Madison (USA) 
6896 OC Taper control of radially symmetric gradient-index waveguides in photopolymer [6896-11]

R. R. McLeod, A. C. Sullivan, Univ. of Colorado at Boulder (USA)

\section{SESSION 4 DIFFRACTIVE PHOTONICS}

6896 OE High efficiency diffractive incouplers for light guides (Invited Paper) [6896-14]

P. Laakkonen, S. Siitonen, Nanocomp Oy Ltd. (Finland) and Univ. of Joensuu (Finland);

T. Levola, Nokia Research Ctr. (Finland); M. Kuittinen, Univ. of Joensuu (Finland)

6896 OF Add-drop filters based on mode conversion Bragg grating cavities [6896-15]

J. B. Khurgin, Johns Hopkins Univ. (USA); M. W. Pruessner, T. H. Stievater, W. S. Rabinovich, Naval Research Lab. (USA)

6896 OG Engineering the spectral response of waveguide Bragg gratings patterned by deep ultraviolet nanolithography [6896-16]

C. M. Greiner, D. lazikov, T. W. Mossberg, LightSmyth Technologies, Inc. (USA); B. McGinnis, R. Narevich, A. Ticknor, NeoPhotonics Corp. (USA)

$68960 \mathrm{H}$ Demultiplexer with blazed waveguide sidewall grating and subwavelength grating structure [6896-17]

P. J. Bock, Univ. of Ottawa (Canada) and National Research Council Canada (Canada);

P. Cheben, J. H. Schmid, D.-X. XU, S. Janz, National Research Council Canada (Canada);

T. J. Hall, Univ. of Ottawa (Canada)

6896 Ol Waveguide Bragg gratings with tailored spectral chirps induced by tapered core profiles [6896-18]

M. Kim, J. J. Ju, S. K. Park, Electronics and Telecommunications Research Institute (South

Korea); M.-H. Lee, Sungkyunkwan Univ. (South Korea)

\section{SESSION $5 \quad$ PLASMONICS}

6896 OK Surface plasmon-polariton mode amplification in long range waveguides [6896-20]

I. De Leon, P. Berini, Univ. of Ottawa (Canada)

6896 OL Gain-induced switching in metal-dielectric-metal plasmonic waveguides [6896-21]

Z. YU, G. Veronis, M. L. Brongersma, S. Fan, Stanford Univ. (USA)

$68960 \mathrm{M}$ Waveguide-ring resonator-based photonic components utilizing channel plasmon polaritons [6896-22]

V. S. Volkov, S. I. Bozhevolnyi, Aalborg Univ. (Denmark)

6896 OP Development of versatile waveguide-coupled optofluidic micro-ring resonator devices [6896-25]

I. M. White, Univ. of Missouri, Columbia (USA); S. Lacey, Franklin \& Marshall College (USA);

J. Gohring, Y. Sun, X. Fan, Univ. of Missouri, Columbia (USA) 
$68960 Q$ Tunable ring resonators for silicon Raman laser and amplifier applications [6896-26]

J. K. Doylend, Intel Corp. (USA); O. Cohen, Intel Corp. (Israel); M. R. Lee, Intel Corp. (USA);

O. Raday, Intel Corp. (Israel); S. Xu, V. Sih, H. Rong, M. Paniccia, Intel Corp. (USA)

6896 OR Waferbonded active/passive vertically coupled microring lasers [6896-27]

M. Hamacher, H. Heidrich, U. Troppenz, Heinrich-Hertz-Institut (Germany); D. Syvridis,

D. Alexandropoulos, S. Mikroulis, A. Kapsalis, Univ. of Athens (Greece); C. W. Tee,

K. Williams, Univ. of Cambridge (United Kingdom); V. Dragoi, EV Group E. Thallner GmbH

(Austria); M. Alexe, Max Planck Institute of Microstructure Physics (Germany); D. Cristea,

M. Kusko, National Institute for Research and Development in Microtechnologies

(Romania)

6896 OS Simulation of integrated coupled nonlinear microring resonators all-optical pulse restorer [6896-28]

Y. Dumeige, L. Ghişa, T. K. N. Nguyên, P. Féron, ENSSAT-FOTON, CNRS-UMR 6082, Univ. de Rennes (France)

\section{SESSION 7 FIBER COMMUNICATION}

6896 OT Noise reduction for fiber optic NTSC signal transmission [6896-29]

R. Furukawa, K. Uehara, Keio Univ. (Japan) and Japan Science and Technology Agency (Japan); S. Takahashi, N. Ohtsu, Japan Science and Technology Agency (Japan); Y. Koike, Keio Univ. (Japan) and Japan Science and Technology Agency (Japan)

6896 OU Cost effective optical coupling for enhanced rate polymer optical fiber communication [6896-30]

J. Chandrappan, J. Zhang, P. V. Ramana, J. H. S. Lau, D. L. Kwong, A*STAR (Singapore)

6896 OV A novel modulation format based on the change of an optical spectrum shape [6896-31]

A. V. Shamray, A. S. Kozlov, I. V. Ilichev, M. P. Petrov, loffe Physico-Technical Institute (Russia)

\section{SESSION $8 \quad$ INTEGRATION CONCEPTS}

6896 OX III-V silicon heterogeneous integration for integrated transmitters and receivers (Invited Paper) [6896-33]

D. Van Thourhout, J. Van Campenhout, G. Roelkens, J. Brouckaert, R. Baets, Ghent Univ. (Belgium)

6896 OY Monolithic growth of the direct band gap material Ga(NAsP) lattice matched on (001) silicon substrate [6896-34]

B. Kunert, NAsPıII/v GmbH (Germany); I. Németh, S. Zinnkann, G. Lukin, T. B. Adams, K. Volz, W. Stolz, Philipps-Univ. Marburg (Germany)

$68960 Z$ Design, fabrication, and integration of HIC glass waveguides on a silicon platform [6896-35] J. Hu, Massachusetts Institute of Technology (USA); N. Carlie, Clemson Univ. (USA); N.-N. Feng, Massachusetts Institute of Technology (USA); L. Petit, Clemson Univ. (USA); A. Agarwal, Massachusetts Institute of Technology (USA); K. Richardson, Clemson Univ. (USA); L. Kimerling, Massachusetts Institute of Technology (USA) 
689611 Hybrid and monolithic integration of planar lightwave circuits (PLCs) (Invited Paper) [6896-37]

R. T. Chen, The Univ. of Texas, Austin (USA)

\section{SESSION 9 MEASUREMENT AND SENSORICS I}

689612 Polymer microring resonators and their sensor applications (Invited Paper) [6896-38] L. J. Guo, A. Maxwell, C.-Y. Chao, T. Ling, J.-S. Kim, S.-W. Huang, S. Ashkenazi, Univ. of Michigan (USA)

689614 Surface enhanced Raman spectroscopy on chip [6896-40]

J. Hubner, T. Anhøj, S. Pedersen, D. A. Zauner, A. M. Jorgensen, G. Blagoi, Technical Univ. of Denmark (Denmark); I. Talian, P.J. Šafárik Univ. (Slovak Republic); O. Hansen, Technical Univ. of Denmark (Denmark)

689615 Miniaturized opto-fluidic ring resonator for sensitive label-free viral detection [6896-41] H. Zhu, I. M. White, J. D. Sutter, Univ. of Missouri, Columbia (USA); M. Zourob, Biophage Pharma Inc. (Canada); X. Fan, Univ. of Missouri, Columbia (USA)

\section{SESSION 10 MEASUREMENT AND SENSORICS II}

689616 Optical field probing in photonic structures by atomic force microscopy combined with optical heterodyne detection (Invited Paper) [6896-42]

S. Blaize, G. Lérondel, A. Bruyant, R. Bachelot, P. Royer, Institut Charles Delaunay, LNIO, Univ. Technologique de Troyes (France)

689617 A compact SWIFTS spectrograph with a leaky loop structure [6896-44]

B. Martin, A. Morand, P. Benech, IMEP-LAHC (France); G. Leblond, S. Blaize, G. Lerondel, LNIO (France); E. Lecoarer, LAOG (France)

689618 Integrated waveguide mixer/splitter for lab-on-a-chip applications [6896-56]

S. Goel, J. N. McMullin, Univ. of Alberta (Canada)

\section{POSTER SESSION}

689619 Innovative design of cassegrain solar concentrator system for indoor illumination utilizing chromatic aberration to filter out ultraviolet and infrared in sunlight [6896-45]

A. J. Whang, B.-Y. Wu, Y.-Y. Chen, National Taiwan Univ. of Science and Technology (Taiwan)

6896 1A Analysis of light guiding property in light piped based solar concentrator [6896-46] A. J. Whang, C.-S. Chuang, Jr., Y.-Y. Chen, National Taiwan Univ. of Science and Technology (Taiwan)

6896 1B Light collection system unit design via mathematical modeling approach [6896-47] A. J. Whang, C.-M. YU, Jr., Y.-Y. Chen, National Taiwan Univ. of Science and Technology (Taiwan) 
6896 1C Innovative design of parabolic reflector light guiding structure [6896-48]

A. J. Whang, C.-H. Tso, Y.-Y. Chen, National Taiwan Univ. of Science and Technology (Taiwan)

$68961 \mathrm{G}$ The modeling of MMI structures for signal processing applications [6896-52]

T. T. Le, L. W. Cahill, La Trobe Univ. (Australia)

$68961 \mathrm{H}$ The use of a radix 5 base for transmission and storage of information [6896-53]

B. S. Tice, Advanced Human Design (USA)

$689611 \quad$ Opto-electronic oscillator: moving toward solutions based on polymer materials [6896-54]

L. D. Nguyên, B. Journet, SATIE, D'Alembert Institute, ENS de Cachan (France); J. Zyss, LPQM, D'Alembert Institute, ENS de Cachan (France)

$68961 \mathrm{~J}$ Improving the beam quality in LMA fibers [6896-55]

E. Voiculescu, Technical Univ. of Cluj Napoca (Romania); M. Hotoleanu, Liekki Oy (Finland);

G. Csipkes, Technical Univ. of Cluj Napoca (Romania)

Author Index 
Downloaded From: https://www.spiedigitallibrary.org/conference-proceedings-of-spie on 26 Apr 2023

Terms of Use: https://www.spiedigitallibrary.org/terms-of-use 


\title{
Conference Committee
}

\author{
Symposium Chair
}

Ali Adibi, Georgia Institute of Technology (USA)

Symposium Cochair

James G. Grote, Air Force Research Laboratory (USA)

Program Track Chair

Yakov Sidorin, Photineer Technology Group (USA)

Conference Chairs

Christoph M. Greiner, LightSmyth Technologies, Inc. (USA)

Christoph A. Waechter, Fraunhofer-Institut für Angewandte Optik und Feinmechanik (Germany)

Conference Cochairs

Jean-Emmanuel Broquin, École Nationale Supérieure d'Electronique et de Radioélectricité de Grenoble (France)

Yakov Sidorin, Photineer Technology Group (USA)

Program Committee

John V. Badding, The Pennsylvania State University (USA)

Trevor M. Benson, The University of Nottingham (United Kingdom)

Pierre Berini, University of Ottawa (Canada)

Xudong Fan, University of Missouri, Columbia (USA)

Helmut Heidrich, Fraunhofer-Institut für Nachrichtentechnik HeinrichHertz-Institut (Germany)

Robert L. Nelson, Air Force Research Laboratory (USA)

Gualtiero Nunzi Conti, Centro Studi e Ricerche Enrico Fermi (Italy) and Istituto di Fisica Applicata Nello Carrara (Italy)

Session Chairs

$1 \quad$ Modeling

Christoph A. Waechter, Fraunhofer-Institut für Angewandte Optik und Feinmechanik (Germany)

2 Glass Waveguides and Devices

Jean-Emmanuel Broquin, École Nationale Supérieure d'Electronique et de Radioélectricité de Grenoble (France) 
3 WG Engineering and Nanofabrication

Robert L. Nelson, Air Force Research Laboratory (USA)

$4 \quad$ Diffractive Photonics

Christoph M. Greiner, LightSmyth Technologies, Inc. (USA)

5 Plasmonics

Pierre Berini, University of Ottawa (Canada)

$6 \quad$ Ring Resonators

Gualtiero Nunzi-Conti, Centro Studi e Ricerche Enrico Fermi (Italy) and Istituto di Fisica Applicata Nello Carrara (Italy)

7 Fiber Communication

Christoph A. Waechter, Fraunhofer-Institut für Angewandte Optik und Feinmechanik (Germany)

$8 \quad$ Integration Concepts

Yakov Sidorin, Photineer Technology Group (USA)

9 Measurement and Sensorics I

Xudong Fan, University of Missouri, Columbia (USA)

10 Measurement and Sensorics II

Jean-Emmanuel Broquin, Ecole Nationale Supérieure d'Electronique et de Radioélectricité de Grenoble (France) 